\title{
THE APPROXIMATE SOLUTION OF DUAL INTEGRAL EQUATIONS BY VARIATIONAL METHODS
}

\author{
by B. NOBLE \\ (Received 15th October 1957)
}

\section{Dual Integral Equations}

The classic application of dual integral equations occurs in connexion with the potential of a circular disc (e.g. Titchmarsh (9), p. 334). Suppose that the disc lies in $z=0,0 \leqslant \rho \leqslant 1$, where we use cylindrical coordinates $(\rho, z)$. Then it is required to find a solution of

$$
\frac{\partial^{2} \phi}{\partial \rho^{2}}+\frac{1}{\rho} \frac{\partial \phi}{\partial \rho}+\frac{\partial^{2} \phi}{\partial z^{2}}=0
$$

such that on $z=0$

$$
\phi=1,(0 \leqslant \rho<1): \partial \phi / \partial z=0,(\rho>1) .
$$

Separation of variables in conjunction with the conditions that $\phi$ is finite on the axis and $\phi$ tends to zero as $z$ tends to plus infinity yields the particular solution $J_{0}(\xi \rho) \exp (-\xi z), z \geqslant 0,0 \leqslant \xi<\infty$. Superposition gives the general solution

$$
\phi=\int_{0}^{\infty} A(\xi) J_{0}(\xi \rho) e^{-\xi z} d \xi,(z \geqslant 0)
$$

In the case of the electrified disc the unknown function $A(\xi)$ is determined by the following dual integral equations obtained by applying the boundary conditions (2):

$$
\begin{aligned}
& \int_{0}^{\infty} A(\xi) J_{0}(\xi \rho) d \xi=1, \quad(0 \leqslant \rho<1), \\
& \int_{0}^{\infty} \xi A(\xi) J_{0}(\xi \rho) d \xi=0, \quad(\rho>1) .
\end{aligned}
$$

The above procedure can be generalised in the following way. Suppose that by separation of variables we can find a solution of a partial differential equation in two variables $x, y$ in the form $X(t, x) Y(t, y)$ where $t$ is a separation parameter. Suppose that the general solution can be obtained by superposition in the form

$$
\phi=\int_{a}^{\beta} A(t) X(t, x) Y(t, y) d t
$$

where $A(t)$ is an arbitrary function. (In the general case $t$ may be complex and the integration in (5) may be over a contour in the complex plane. If we are dealing with a region possessing discrete eigenvalues, (5) is replaced by

$$
\phi=\sum_{n} A_{n} \dot{X}\left(t_{n}, x\right) Y\left(t_{n}, y\right),
$$


where the $t_{n}$ are the permissible values of $t$. In either of these cases the theory can be carried through as for (5)).

Suppose that by suitable choice of $X, Y$ we can satisfy all boundary conditions except on $y=y_{0}$ where

$$
\phi=f(x),(a \leqslant x<c): \partial \phi / \partial y=g(x),(c<x \leqslant b) .
$$

Then from (5)

$$
\begin{aligned}
& \int_{a}^{\beta} M(t) X(t, x) A(t) d t=f(x),(a \leqslant x<c), \\
& \int_{a}^{\beta} N(t) X(t, x) A(t) d t=g(x),(c<x \leqslant b),
\end{aligned}
$$

where we have written

$$
M(t)=Y\left(t, y_{0}\right): N(t)=[\partial Y(t, y) / \partial y]_{y=y_{0}} .
$$

These are dual integral equations which determine the unknown function $A(t)$.

\section{Reduction to a Single Integral Equation}

Assume that there is an inversion formula

$$
\begin{aligned}
& \int_{a}^{\beta} X(t, x) \beta(t) d t=b(x), \quad(a \leqslant x \leqslant b), \\
& \int_{a}^{b} X(t, x) b(x) d x=\beta(t), \quad(\alpha \leqslant t \leqslant \beta) .
\end{aligned}
$$

Suppose that the left-hand side of $(6 b)$ equals the (unknown) function $u(x)$ for $a \leqslant x<c$. Inversion gives

$$
N(t) A(t)=\int_{a}^{c} X(t, \xi) u(\xi) d \xi+\int_{c}^{b} X(t, \xi) g(\xi) d \xi .
$$

The substitution of this expression for $A(t)$ in $(6 a)$ gives an integral equation for $u(x)$, valid in $a \leqslant x<c$ :

$$
\begin{aligned}
\int_{a}^{\beta} \frac{M(t)}{N(t)} X(t, x) \int_{a}^{c} X(t, \xi) u(\xi) d \xi d t & \\
& =f(x)-\int_{a}^{\beta} \frac{M(t)}{N(t)} X(t, x) \int_{c}^{b} X(t, \xi) g(\xi) d \xi d t .
\end{aligned}
$$

An alternative procedure is to assume that the left-hand side of $(6 a)$ equals the (unknown) function $v(x)$ for $c<x \leqslant b$. As above we can invert to find $A(t)$. Substitution in $(6 b)$ then gives an integral equation in $c<x \leqslant b$ for $v(x)$ :

$$
\begin{aligned}
\int_{a}^{\beta} \frac{N(t)}{M(t)} X(t, x) \int_{c}^{b} X(t, \xi) v(\xi) d \xi d t & \\
& =g(x)-\int_{a}^{\beta} \frac{N(t)}{M(t)} X(t, x) \int_{a}^{c} X(t, \xi) f(\xi) d \xi d t .
\end{aligned}
$$




\section{SOLUTION OF DUAL INTEGRAL EQUATIONS}

If it is permissible to interchange orders of integration on the left of (8), (9), this would give standard integral equations of the first kind with the following kernels :

$$
\begin{aligned}
& K_{1}(x, \xi)=\int_{a}^{\beta} \frac{M(t)}{N(t)} X(t, x) X(t, \xi) d t, \\
& K_{2}(x, \xi)=\int_{a}^{\beta} \frac{N(t)}{M(t)} X(t, x) X(t, \xi) d t .
\end{aligned}
$$

Unfortunately it will be found in concrete examples that one of these integrals is divergent. (This is easily verified for equations (4) which are typical.) Various manipulations can be used to remove the divergency but it will not be necessary to consider these since the variational principles of $\S 3$ apply directly to (8) and (9).

\section{Basic Variational Principles}

A fundamental variational principle for integral equations is the following. Suppose that $e(x)$ is a solution of

$$
\lambda e(x)+\int_{p}^{q} K(x, \xi) e(\xi) d \xi=w(x), \quad(p \leqslant x \leqslant q),
$$

where all the functions are real, $K(x, \xi)$ is symmetrical, and $\lambda$ is a given constant which may be zero. Then the expression

$$
J(E)=\int_{p}^{q}\left\{2 w(x) E(x)-\lambda E^{2}(x)\right\} d x-\int_{p}^{q} \int_{p}^{q} K(x, \xi) E(x) E(\xi) d x d \xi
$$

has a stationary value when $E(x)$ varies round $e(x)$, the solution of (11).

For the purposes of this paper we shall consider, instead of (12),

$$
J(E)=\int_{p}^{q}\left\{2 v(x) E(x)-\lambda E^{2}(x)\right\} d x-\int_{\beta}^{a} P(t) T^{2}(t) d t,
$$

where

$$
T(t)=\int_{p}^{q} X(t, x) E(x) d x
$$

Set

$$
E(x)=e(x)+\epsilon \eta(x),
$$

where $\eta(x)$ is an arbitrary function and $\epsilon$ is a parameter. If (14) is substituted in (13) we find

$$
J(E)=J(e)+2 \epsilon I(e, \eta)-\epsilon^{2}\left\{\lambda \int_{p}^{q} \eta^{2}(x) d x+\int_{a}^{\beta} P(t) N^{2}(t) d t\right\},
$$

where

$$
N(t)=\int_{p}^{q} X(t, x) \eta(x) d x
$$

$$
I(e, \eta)=\int_{p}^{q}\left\{w(x)-\lambda e(x)-\int_{a}^{\beta} P(t) X(t, x) \int_{a}^{\beta} X(t, \xi) e(\xi) d \xi\right\} \eta(x) d x .
$$

In this last expression we have assumed that orders of integration in $x$ and $t$ 
can be interchanged. $J(E)$ defined in (13) is stationary with reference to variations of $E(x)$ about $e(x)$ if the coefficient of $\epsilon$ in (15) is zero for arbitrary $\eta(x)$, i.e. from (16), if

$$
\lambda e(x)+\int_{a}^{\beta} P(t) X(t, x) \int_{p}^{q} X(t, \xi) e(\xi) d \xi d t=w(x), \quad(p \leqslant x \leqslant q) .
$$

This is an integral equation for $e(x)$ but in one way it is more general than (11) since it is only when orders of integration in $\xi$ and $t$ in (17) can be interchanged that (17) can be reduced to (11) with (cf. $(10 a, b)$ :

$$
K(x, \xi)=\int_{a}^{\beta} P(t) X(t, x) X(t, \xi) d t .
$$

From (15), if $e(x)$ satisfies (17), $P(t) \geqslant 0(\alpha \leqslant t \leqslant \beta)$, and $\lambda \geqslant 0$, then

$$
J(e) \geqslant J(E) \text {. }
$$

It is easily shown that

$$
J(e)=\int_{p}^{q} w(x) e(x) d x
$$

In physical applications it will appear that this quantity has invariably a direct physical significance. It is usually connected with the total energy of the system. that

In order to apply these results we proceed in the usual way by assuming

$$
e(x) \bumpeq E(x)=\sum_{k=1}^{n} k_{i} \psi_{i}(x)
$$

where the $\psi_{i}(x)$ are known functions and the $k_{i}$ are constants to be determined from the condition that (13) is stationary. On substituting (20) in (13) and setting $d J / d k_{i}=0,(i=1$ to $n)$, the following set of simultaneous linear algebraic equations is found for the $k_{i}$ :

$$
\sum_{j=1}^{n} \Delta\left(\psi_{i}, \psi_{j}\right) k_{j}=\left[w, \psi_{i}\right], \quad(i=1 \text { to } n)
$$

where

$$
[w, \psi]=\int_{p}^{q} w(x) \psi(x) d x,
$$

$$
\begin{aligned}
\Delta(\chi, \psi)=\lambda \int_{p}^{q} \chi(x) \psi(x) d x & \\
& \quad+\int_{a}^{\beta} P(t) \int_{p}^{q} X(t, x) \chi(x) d x \int_{p}^{q} X(t, \xi) \psi(\xi) d \xi d t .
\end{aligned}
$$

Obviously the calculations will be simplified if the $\psi_{i}(x)$ are chosen so that

$$
\int_{p}^{q} X(t, x) \psi_{i}(x) d x
$$

can be evaluated explicitly. 
In practice it often happens that we can guess the shape of $e(x)$ reasonably accurately, e.g. by physical intuition, and we need use only one term of the series (20). If we set $e(x) \bumpeq k \psi(x)$, a straightforward calculation gives

$$
k=\frac{[w, \psi]}{\Delta(\psi, \psi)} \quad: \quad J(E)=\frac{[w, \psi]^{2}}{\Delta(\psi, \psi)}
$$

It is expressions of this form which usually appear in Schwinger's applications of the variational method.

\section{Upper and Lower Limits}

In $\$ 2$ we reduced our dual integral equations to a single integral equation in two different ways. The variational principle in $\$ 3$ can be applied to each of these in turn. Suppose that $U(x), V(x)$ are approximations to the solutions $u(x), v(x)$ of $(8),(9)$. Define

$$
\begin{aligned}
& R(t)=\int_{a}^{c} X(t, x) U(x) d x: S(t)=\int_{c}^{b} X(t, x) V(x) d x \\
& F(t)=\int_{a}^{c} X(t, x) f(x) d x: G(t)=\int_{c}^{b} X(t, x) g(x) d x .
\end{aligned}
$$

From (13) the following quantities are stationary with respect to variations in $U, V$ :

$$
\begin{array}{r}
H(U)=2 \int_{a}^{c} f(x) U(x) d x-2 \int_{a}^{\beta} \frac{M(t)}{N(t)} R(t) G(t) d t \\
\quad-\int_{a}^{\beta} \frac{M(t)}{N(t)} R^{2}(t) d t, \ldots \\
I(V)=2 \int_{c}^{b} g(x) V(x) d x-2 \int_{a}^{\beta} \frac{N(t)}{M(t)} S(t) F(t) d t \\
-\int_{a}^{\beta} \frac{N(t)}{M(t)} S^{2}(t) d t .
\end{array}
$$

When $U, V$ are identical with $u, v$, inversion of $(6 a, b)$ gives

$$
M(t) A(t)=S(t)+F(t): N(t) A(t)=R(t)+G(t) .
$$

Then

$$
\begin{aligned}
H(u) & =\int_{a}^{c} f(x) u(x) d x-\int_{c}^{b} g(x) v(x) d x+\int_{a}^{\beta} \frac{M(t)}{N(t)} G^{2}(t) d t, \\
I(v) & =\int_{c}^{b} g(x) v(x) d x-\int_{a}^{c} f(x) u(x) d x+\int_{a}^{\beta} \frac{N(t)}{M(t)} F^{2}(t) d t .
\end{aligned}
$$

From (18),

E.M.S.-H

$$
H(u) \geqslant H(U): I(v) \geqslant I(V)
$$


Hence

$$
\begin{aligned}
\mathbf{H}(U)-\int_{a}^{\beta} \frac{M(t)}{N(t)} G^{2}(t) d t \leqslant \int_{a}^{c} f(x) u(x) d x- & \int_{c}^{b} g(x) v(x) d x \\
& \leqslant \int_{a}^{\beta} \frac{N(t)}{M(t)} F^{2}(t) d t-I(V) .
\end{aligned}
$$

It is sometimes convenient to express this basic inequality in a different way. Introduce $\Lambda(U), \Gamma(U)$ defined by

$$
\Lambda(U)=\frac{Q_{1}-H(U)}{H(U)-Q_{2}}: \Gamma(V)=\frac{Q_{1}-I(V)}{I(V)-Q_{2}}
$$

where $Q_{1}, Q_{2}$ are known constants given by

$$
Q_{1}=\int_{a}^{\beta} \frac{N(t)}{M(t)} F^{2}(t) d t: Q_{2}=\int_{a}^{\beta} \frac{M(t)}{N(t)} G^{2}(t) d t .
$$

Let $\Lambda(u), \Gamma(v)$ denote the corresponding quantities with $U, V$ replaced by $u, v$. From (27),

Hence

$$
H(u)-Q_{2}=Q_{1}-I(v)
$$

$$
\Lambda(u)=\{\Gamma(v)\}^{-1}=D(u, v), \text { say }
$$

From (28), (30),

Finally, therefore,

$$
\Lambda(u) \leqslant \Lambda(U): \Gamma(v) \leqslant \Gamma(V)
$$

$$
\Lambda(U) \geqslant D(u, v) \geqslant\{\Gamma(V)\}^{-1} \text {. }
$$

The point of these manipulations appears most clearly when either $f(x)$ or $g(x)$ is identically zero. Suppose that $g(x) \equiv 0$. The expressions $(25 a, b)$ for $H(U), I(V)$, and the inequalities (29) lose their formal symmetry. But if $U, V$ are replaced by $k U, l V$ and the optimum $k, l$ are found as in (18), (19) we obtain

$$
\begin{aligned}
H(k U) & =\left\{\int_{a}^{\beta} R(t) F(t) d t\right\}^{2}\left\{\int_{a}^{\beta} \frac{M(t)}{N(t)} R^{2}(t) d t\right\}^{-1}, \ldots . \\
I(l V) & =\left\{\int_{a}^{\beta} \frac{N(t)}{M(t)} S(t) F(t) d t\right\}^{2}\left\{\int_{a}^{\beta} \frac{N(t)}{M(t)} S^{2}(t) d t\right\}^{-1} .
\end{aligned}
$$

In the first equation we have used the result

$$
\int_{a}^{c} f(x) U(x) d x=\int_{a}^{\beta} R(t) F(t) d t .
$$

$(33 a, b)$ have formal symmetry if we consider that $R(t)$ corresponds to

$$
N(t) S(t)\{M(t)\}^{-1} \text {. }
$$

The inequalities (29) are asymmetrical but $\Lambda(k U), \Gamma(k V)$ are now symmetrical so that the inequalities (32) are symmetrical. 


\section{Upper and Lower Limits (continued)}

In the last section, in order to obtain both upper and lower limits it was necessary to guess the form of two unknown functions $U(x)$ and $V(x)$. We now show that it is sufficient to guess the form of only one of these functions.

For simplicity we assume $g(x)=0$. Suppose that we guess the form of $U(x)(a \leqslant x<c)$. By inversion of $(6 b)$ an approximate expression for $A(t)$ is given by

$$
\{N(t)\}^{-1} \int_{a}^{c} X(t, z) U(z) d z .
$$

Substitution in $(6 a)$ gives, for $c<x<b$,

$$
V(x)=\int_{a}^{\beta} \frac{M(t)}{N(t)} X(t, x) \int_{a}^{c} X(t, z) U(z) d z d t .
$$

If this expression for $V(x)$ is substituted in $S(t)$, defined in $(24 a)$, we find

$$
S(t)=\frac{M(t)}{\bar{N}(t)} R(t)-W(t)
$$

where $R(t)$ is defined in $(24 a)$ and

$$
W(t)=\int_{a}^{c} X(t, x) \int_{a}^{\beta} \frac{M(\xi)}{N(\xi)} X(\xi, x) R(\xi) d \xi d x .
$$

We have

$$
\int_{a}^{\beta} R(t) W(t) d t=\int_{a}^{\beta} \frac{M(t)}{N(t)} R^{2}(t) d t .
$$

On using these results we find after some manipulation that if $V(x)$ is chosen as in (34),

$$
\int_{a}^{\beta} \frac{N(t)}{M(t)} F^{2}(t) d t-I(V)=H(U)+\int_{a}^{\beta} \frac{N(t)}{M(t)}\{F(t)-W(t)\}^{2} d t .
$$

Hence (29) becomes

$$
H(U) \leqslant \int_{a}^{c} f(x) u(x) d x \leqslant H(U)+\int_{a}^{\beta} \frac{N(t)}{M(t)}[F(t)-W(t)]^{2} d t .
$$

If we define the error $\Delta(x)$ in our solution of the original integral equation (8) by

$$
\Delta(x)=\int_{a}^{\beta} \frac{M(t)}{N(t)} X(t, x) \int_{a}^{c} X(t, \xi) U(\xi) d \xi d t-f(x),
$$

then from (35) and $(24 b)$,

$$
W(t)-F(t)=\int_{a}^{c} X(t, z) \Delta(x) d x .
$$

This shows in a direct way that if $U(x)$ is a good approximation to the solution of the integral equation then the difference between the upper and lower limits in (36) will be small. This also indicates a possible practical method of approach to the evaluation of the error term. 


\section{Application to Parallel Discs}

As a first application consider the potential due to two parallel discs lying in $z= \pm d, 0 \leqslant p<1$. If the two discs are at the same potential we find

$$
\begin{aligned}
\phi & =\int_{0}^{\infty} B(\xi) J_{0}(\xi \rho) e^{-\xi|z|} d \xi, \quad(|z| \geqslant d), \ldots \\
& =\int_{0}^{\infty} C(\xi) J_{0}(\xi \rho) \cosh \xi z d \xi, \quad(|z| \leqslant d) .
\end{aligned}
$$

Continuity of potential on $z=d$ gives

$$
B(\xi) e^{-\xi d}=C(\xi) \cosh \xi d=A(\xi)\left(1+e^{-2 \xi d}\right), \text { say. }
$$

If the dises are at unit potential the boundary conditions on $z=d$ are

$$
\phi=1,(0 \leqslant \rho<1):(\partial \phi / \partial z) \text { continuous for } \rho>1 \text {. }
$$

These give the following dual integral equations :

$$
\begin{aligned}
& \int_{0}^{\infty}\left(1+e^{-2 \xi d}\right) A(\xi) J_{0}(\xi \rho) d \xi=1, \quad(0 \leqslant \rho<1), \\
& \int_{0}^{\infty} \xi A(\xi) J_{0}(\xi \rho) d \xi=0, \quad(\rho>1) . \quad \ldots \ldots \ldots \ldots \ldots . . .
\end{aligned}
$$

If the two discs are at opposite potentials the $\cosh \xi z$ in $(39 b)$ is replaced by $\sinh \xi z$ and we find the same dual integral equations (41) except that in (41a) the term $\{1+\exp (-2 \xi d)\}$ is replaced by $\{1-\exp (-2 \xi d)\}$.

If we assume that the right-hand side of $(41 b)$ equals the (unknown) function $u(\rho)$ for $0 \leqslant \rho<1$, then the theory of $\S \S 2,3$ shows that the following expression is stationary when $U(r)$ varies round $u(r)$ :

$$
H(U)=2 \int_{0}^{1} r U(r) d r-\int_{0}^{\infty}\left(1 \pm e^{-2 \xi d}\right) R^{2}(\xi) d \xi
$$

where

$$
R(\xi)=\int_{0}^{1} r J_{0}(\xi r) U(r) d r
$$

The upper and lower signs refer to dises at the same and opposite potentials respectively. We have

$$
H(u)=\int_{0}^{1} r u(r) d r .
$$

From $(39 b),(40),(41 b)$, for $0 \leqslant \rho<1$,

$$
u(\rho)=\int_{0}^{\infty} \xi A(\xi) J_{0}(\xi \rho) d \xi=\frac{1}{2}\left\{\left(\frac{\partial \phi}{\partial z}\right)_{d-0}-\left(\frac{\partial \phi}{\partial z}\right)_{d+0}\right\}=2 \pi \sigma(\rho),
$$

where $\sigma(\rho)$ is the charge density on the disc and electrostatic units are used. Hence the total charge on a single disc is given by

$$
Q=2 \pi \int_{0}^{1} r \sigma(r) d r=\int_{0}^{1} r u(r) d r .
$$

The quantity $H(U)$ in (42) therefore gives an estimate of the charge on a single disc. 
We first of all choose

$$
U(\rho)=k\left(1-\rho^{2}\right)^{-\rightarrow},(0 \leqslant \rho<1),
$$

where $k, \nu$ are constants to be determined by the stationary character of (42). Then

$$
R(\xi)=k \int_{0}^{1} r\left(1-r^{2}\right)^{-v} J_{0}(\xi r) d r=k 2^{-v} \Gamma(1-v) t^{\nu-1} J_{1-\nu}(t),
$$

where we have used Sonine's first finite integral, ((10), p. 373). Substitution in (42) and determination of the optimum $k$ as for (22) gives

$$
\{H(U)\}^{-1}=2^{2-2 \nu}\{\Gamma(2-\nu)\}^{2}\left\{D_{\nu}\left((0) \pm D_{\nu}(2 d)\right\},\right.
$$

TABLE I

\begin{tabular}{|c|c|c|c|c|c|}
\hline & $d$ & 0.2 & 0.5 & $1 \cdot 0$ & $2 \cdot 5$ \\
\hline \multirow{2}{*}{$\begin{array}{c}\text { Same } \\
\text { potential }\end{array}$} & $(47 a)$ & 0.5999 & 0.6903 & 0.7816 & 0.88958 \\
\cline { 2 - 6 } & Nomura & 0.6027 & 0.6912 & 0.7822 & 0.88958 \\
\hline \multirow{2}{*}{$\begin{array}{c}\text { Opposite } \\
\text { potentials }\end{array}$} & $(47 a)$ & 3.0023 & $1 \cdot 8138$ & $1 \cdot 3876$ & $1 \cdot 14172$ \\
\cline { 2 - 6 } & Nomura & $3 \cdot 1029$ & $1 \cdot 8208$ & $1 \cdot 3867 *$ & $1 \cdot 14172$ \\
\hline
\end{tabular}

where

$$
D_{\nu}(q)=\int_{0}^{\infty} e^{-q t}\left\{t^{\nu-1} J_{1-\nu}(t)\right\}^{2} d t .
$$

Physically it is clear that for parallel discs at the same potential the charge distribution on the discs will be approximately the same as that for an isolated. disc. This will also hold for discs at opposite potentials providing that the discs are far apart. In these cases it is reasonable to choose $\nu=\frac{1}{2}$ in (46) and we find

$$
\left\{\frac{1}{2} \pi H(U)\right\}^{-1}=\left\{1 \pm D_{\frac{1}{2}}(2 d)\right\}
$$

where

$$
D_{\frac{1}{2}}(2 d)=(2 / \pi)\left\{\arctan \left(d^{-1}\right)-\frac{1}{2} d \ln \left(1+d^{-2}\right)\right\} .
$$

Table I compares $\frac{1}{2} \pi H(U)$ calculated from $(47 a)$ with more exact values obtained by a completely different method in Y. Nomura (7). (The value marked with an asterisk would seem to be in error.)

The accuracy clearly deteriorates for oppositely charged dises when $d$ decreases, as we should expect. In this case a better approximation is to assume a uniform charge distribution on the plates, i.e. $\nu=0$ in (46). Then

$$
\left\{\frac{1}{2} \pi H(U)\right\}^{-1}=8 \pi^{-1}\left\{D_{0}(0)-D_{0}(2 d)\right\} \text {, }
$$

E.M.S.-H 2 
where

$$
\begin{gathered}
D_{0}(2 d)=-d+\frac{4}{3 \pi k^{2}}\left\{\left(1-k^{2}\right) K(k)+\left(2 k^{2}-1\right) E(k)\right\}, \\
D_{0}(0)=4(3 \pi)^{-1}: k=\left(1+d^{2}\right)^{-\frac{1}{2}} .
\end{gathered}
$$

This result has been given in ((8), p. 81) from a different though related point of view.

In the transition region between large $d$ in (47a) (lower sign), and small $d$ in $(48 a)$, we can take

$$
U(p)=C_{1}\left(1-p^{2}\right)^{-\mathbf{t}}+C_{2}
$$

Substitution in (42) gives

$$
H(U)=\alpha C_{1}+\beta C_{2}-a C_{1}^{2}-2 b C_{1} C_{2}-c C_{2}^{2},
$$

where $\alpha=2, \beta=1$, and

$$
\begin{aligned}
& a=\int_{0}^{\infty}\left(1-e^{-2 \xi d}\right)\left(\frac{\sin \xi}{\xi}\right)^{2} d \xi: c=\int_{0}^{\infty}\left(1-e^{-2 \xi d}\right)\left(\frac{J_{1}(\xi)}{\xi}\right)^{2} d \xi \\
& b=I(0)-I(2 d), \\
& I(q)=\int_{0}^{\infty} e^{-q \xi}\left(\frac{\sin \xi}{\xi}\right)\left(\frac{J_{1}(\xi)}{\xi}\right) d \xi \\
& =\frac{1}{2}\left\{\arcsin \left(p-\frac{1}{2} q\right)+q^{\frac{3}{2}}\left(p-\frac{1}{2} q\right)^{\frac{1}{2}}+q^{\frac{1}{4}}\left(p+\frac{1}{2} q\right)^{\frac{1}{4}}\right\}-q, \\
& p=\left(1+\frac{1}{4} q^{2}\right)^{ \pm} .
\end{aligned}
$$

If we define

$$
A=\beta a-\alpha b: B=\alpha c-\beta b: \Delta=a c-b^{2},
$$

it is found on minimising (50) that

$$
C_{1}=(2 \Delta)^{-1} B: C_{2}=(2 \Delta)^{-1} A,
$$

and the minimum is given by

$$
H_{12}=\frac{\alpha^{2}}{4 a}+\frac{A^{2}}{4 a \Delta}=\frac{\beta^{2}}{4 c}+\frac{B^{2}}{4 c \Delta} .
$$

Set

$$
H_{1}=(4 a)^{-1} \alpha^{2}: H_{2}=(4 c)^{-1} \beta^{2} .
$$

These values of $H$ correspond to $C_{2}=0$, i.e. (47), and $C_{1}=0$, i.e. (48), respectively. The additional terms in (51) provide corrections for the first order results and it is convenient to use these forms in numerical work. The results in table II have been obtained from the above formulæ.

Another method for obtaining results which are more accurate than (47) or (48) is to consider general $\nu$ in (46). For small $q$ we can write ((10), p. 403),

$$
\begin{aligned}
D_{\nu}(q) & =\frac{1}{2} \sum_{m=0}^{\infty} \frac{(-1)^{m} 2^{2 \nu+m-1} q^{m} \Gamma(2-2 \nu-m) \Gamma\left(\frac{1}{2}+\frac{1}{2} m\right)}{m !\left\{\Gamma\left(\frac{3}{2}-\nu-\frac{1}{2} m\right)\right\}^{2} \Gamma\left(\frac{5}{2}-2 \nu-\frac{1}{2} m\right)} \\
& +\frac{1}{2} \sum_{m=0}^{\infty} \frac{(-1)^{m} 2^{m+1} q^{2-2 \nu+m} \Gamma(2 \nu-2-m) \Gamma\left(3-\nu+\frac{1}{2} m\right)}{\left.m !\left\{\Gamma \frac{1}{2}-\frac{1}{2} m\right)\right\}^{2} \Gamma\left(\frac{3}{2}-\nu-\frac{1}{2} m\right)}
\end{aligned}
$$


table III has been compiled by using this formula for $\nu=-\frac{1}{4}, \frac{1}{4}, \frac{3}{4}$, and by taking the values for $\nu=0, \frac{1}{2}$, from table II.

The results in tables II and III show clearly that for parallel plates at opposite potentials a constant charge distribution is appropriate when $d<0.05$,

TABLE II

\begin{tabular}{|c|c|c|c|c|}
\hline$d$ & 0.05 & 0.10 & 0.20 & 0.40 \\
\hline$(\pi / 2) H_{1}$ & 7.862 & 4.755 & 3.002 & $2 \cdot 023$ \\
\hline$(\pi / 2) \mathrm{H}_{2}$ & 8.855 & 4.795 & 2.699 & 1.390 \\
\hline$(\pi / 2) H_{12}$ & $9 \cdot 158$ & 5.080 & 3.072 & $2 \cdot 026$ \\
\hline $4 C_{1}$ & 1.82 & 1.56 & 1.40 & 1.22 \\
\hline $4 C_{2}$ & 8.00 & 3.35 & $1 \cdot 15$ & 0.14 \\
\hline
\end{tabular}

TABLE III

\begin{tabular}{|c|c|c|c|}
\hline$d$ & 0.05 & $0 \cdot 10$ & 0.20 \\
\hline$\frac{3}{4}$ & 4.03 & 2.93 & $2 \cdot 16$ \\
\hline$\frac{1}{2}$ & $7 \cdot 86$ & $4 \cdot 76$ & 3.00 \\
\hline$\frac{1}{4}$ & 9.09 & $5 \cdot 13$ & 3.02 \\
\hline 0 & 8.86 & 4.80 & $2 \cdot 70$ \\
\hline$-\frac{1}{4}$ & $8 \cdot 35$ & 4.56 & $2 \cdot 66$ \\
\hline
\end{tabular}

whereas the charge distribution of an isolated disc is more suitable when $d>0 \cdot 2$.

All the results in this section refer to lower limits for the total charge. Numerical results for upper limits will be discussed in a later paper.

\section{Concluding Remarks}

Variational methods were applied extensively to the integral equation formulation of electromagnetic problems by J. Schwinger and his collaborators during the last war. An account of this type of application is given in (1). Other applications are given in (6). The methods used here are related to those of Schwinger but there are two important differences in detail : 
(a) In Schwinger's method the integral equations are formulated by means of Green's functions, the two equivalent integral equations of $\$ 3$ are obtained separately, and often the kernel of the integral equation has to be transformed before convenient formulæ are obtained. The present method deduces the two integral equations in a symmetrical way by using merely separation-ofvariables (or transform methods if preferred). The kernels of the integral equations appear automatically in forms which are convenient for the variational principle.

(b) In Schwinger's method (23) is regarded as the basic variational expression whereas we use (13). The two are equivalent but it is somewhat easier to use (13) in connection with the general expansion (20) and the general theory for upper and lower limits in $\$ \S 4,5$. (In this connection the reader may compare the treatment of upper and lower limits for special examples given in (1), (3), (5)). As already pointed out, (13) avoids divergence difficulties on which various authors have commented (e.g. (2), p. 186, footnote).

In the particular example treated in $\$ 6$ the dual integral equations can be solved iteratively, or they can be reduced to a Fredholm integral equation of the second kind (e.g. (4) and references there given). I have done a considerable amount of work along these lines since 1951 and have come to the conclusion that for general purposes the variational method given in this paper is to be preferred. The other methods are convenient for certain problems but they depend on special properties of Bessel functions. In the examples of $\$ 6$ they are not convenient for the parallel plate condenser with small separation of the plates.

The methods used in this paper can be extended to deal with unsymmetrical kernels, complex kernels, simultaneous integral equations, and estimation of unknown functions at a point instead of estimation of overall parameters.

\section{REFERENCES}

(1) F. E. Borgnis and C. H. PaPas, Randwertprobleme der Mikrowellenphysik, (Springer-Verlag, Berlin, 1955).

(2) B. B. BAKER and E. T. Copson, Huyghens' Principle (Oxford University Press, 1950). 1465 .

(3) J. F. Carlson and T. J. Hendrickson, J. Appl. Phys., 24 (1953), pp. 1462 -

(4) J. C. Cooke, Quart. J. Mech. and Appl. Math., 9 (1956), pp. 103-110.

(5) H. Levine and C. H. Papas, J. Appl. Phys., 22 (1951), pp. 29-43.

(6) P. M. Morse and H. Fesmbach, Methods of Theoretical Physics (MeGrawHill, New York, 1953).

(7) Y. Nomura, Proc. Phys. Math. Soc. Japan, (3) 23, (1941), pp. 168-180.

(8) G. Poly a and G. Szegö, Isoperimetric inequalities in Mathematical Physics, Annals of Math. Studies No. 27, (Princeton University Press, 1951).

(9) E. C. Titchmarsh, Fourier Integrals (Oxford University Press, 1937).

(10) G. N. Watson, Bessel Functions (Cambridge University Press, 1944).

\section{Department of Mathematics}

The Royal College of Science and Technology

GLasgow 